\title{
The treatment of hypertension in people with dementia: a systematic review of observational studies
}

\author{
Tomas J Welsh*, John R Gladman and Adam L Gordon
}

\begin{abstract}
Background: Hypertension is very common in older people and a number of trials of antihypertensives have demonstrated benefit from treatment in even the oldest old. However, people with dementia were significantly under-represented in these studies and as a population are more likely to be physically frail, to suffer orthostatic hypotension and to experience adverse effects from polypharmacy at a lower drug count. It may be that different thresholds for commencement and cessation of treatment should be considered and may already be used for this group. Against this background this review sets out to describe the prevalence of hypertension in people with dementia, its treatment, change in treatment over time and the achievement of blood pressure (BP) control.

Methods: The PubMed, Cochrane, Embase and PsychINFO databases were searched for observational studies involving people with dementia and a diagnosis of hypertension. The search was limited to English language articles involving adults and humans published from 1990 onwards. Abstracts and titles were then reviewed with eligible articles read in full. Bibliographies were examined for further relevant studies. The final selection of studies was then analysed and appraised.
\end{abstract}

Results: Thirteen articles were identified for analysis. The prevalence of hypertension in people with dementia was 45\% (range 35\%-84\%). $73 \%$ of these were on at least one antihypertensive, with diuretics being the most common. The reported prevalence of hypertension in study populations remained unchanged over time. ACEi/ARBs and calcium channel blockers were prescribed more frequently in more recent studies whilst use of $\beta$-blockers and diuretics remained unchanged. Target blood pressure was achieved in 55\% of those on treatment.

Conclusion: Hypertension is as common in people with dementia as in other populations and is as commonly treated with antihypertensive drugs. The findings presented here will support further work to establish the risk-benefit of antihypertensive treatment in patients with dementia and, if differing ratios are identified, to establish dementia-specific guidelines for management.

Keywords: Hypertension, Dementia, Systematic review, Treatment, Antihypertensive

\section{Background}

Hypertension is common in very old people, approximately $80 \%$ of those aged over 80 are hypertensive [1], and remains a risk factor for cardiovascular and cerebrovascular disease in later life. A number of trials of antihypertensive medication, most notably the Hypertension in the Very Elderly Trial (HYVET) [2], the Systolic Hypertension in Europe Study (Syst-Eur) [3], the Systolic

\footnotetext{
* Correspondence: tomas.welsh@nottingham.ac.uk

Division of Rehabilitation and Ageing, School of Medicine, B99, B Floor, University of Nottingham, Nottingham NG7 2UH, UK
}

Hypertension in the Elderly Program (SHEP) [4] and the Study on Cognition and Prognosis in the Elderly (SCOPE) [5], demonstrated that antihypertensives can benefit the oldest old. A Cochrane review of the treatment of hypertension in older adults confirmed that treatment reduced cardiovascular events, but also showed that it was associated with a significant increase in the rate of treatment withdrawal [6].

The findings of these trials have been incorporated into many widely accepted guidelines for the treatment of hypertension, such as those of the Joint National 
Committee on Prevention, Detection, Evaluation, and Treatment of High Blood Pressure (JNC), which have promoted increasingly aggressive targets for blood pressure control in older people [7,8]. Even tighter targets for those with comorbidities, such as diabetes or stroke disease, mean that even lower blood pressure targets are identified for older people in whom such co-morbidities are frequently present. However the average trial patient bears little resemblance to many very old people, in whom the likelihood of treatment withdrawal and hence harm, may be higher. The HYVET study, for instance, involved a physiologically robust group, with a low rate of orthostatic hypotension and no cognitive impairment [2].

People with cognitive impairment are more likely to be physically frail [9], are more likely to suffer orthostatic hypotension [10] and with age-related and arteriosclerotic changes to cerebral blood flow auto-regulation are more vulnerable to resulting cerebral hypoperfusion [11]. In addition they experience adverse events associated with polypharmacy at a lower drug count [12]. It may be, therefore, that the risk-benefit ratio of antihypertensive treatment is different in this cohort. Differing thresholds might be adopted for commencement or cessation of treatment. Many doctors believe this intuitively to be the case. Morley recently questioned whether the findings of key trials in very old people are truly generalizable, concluding that they do not apply to frail very old people and singled out blood pressure as an area where treatment targets should be attenuated [13].

Against this background we set out to describe how hypertension is currently treated in patients with dementia, whether doctors follow generic guidelines or whether they adapt their practice in light of the above considerations.

\section{Methods}

\section{Search strategy and selection criteria}

A pre-specified protocol was used to search for and identify suitable articles.

\section{Eligibility}

Study characteristics: Observational studies of a population with dementia describing the prevalence of hypertension and treatments used.

Report characteristics: Non-English Language articles and studies carried out prior to 1990 were excluded.

\section{Information sources}

A systematic search of the literature was conducted by searching electronic databases, and scanning reference lists of articles. The following databases were used: PubMed (1946 - present), Cochrane, Embase (1974 - present) and PsychINFO (1806 - present). The last full search was run on the 14th of November 2012, with updates to this until April 2013.

\section{Search}

The following groupings of search terms were used and were adapted, as appropriate, for each database: dementia; demented; dementing; hypertension; blood pressure; antihypertensive; hypertensive; treatment; management.

An example search strategy is provided in Additional file 1 .

The search was then limited to English language articles, to studies involving humans and to studies involving adults.

\section{Study selection}

The title and abstract of the retrieved records were then assessed against the eligibility criteria in a standardised manner. Where there was uncertainty about eligibility the full article was reviewed. The bibliographies of eligible articles were searched for further relevant articles, which were again appraised against eligibility criteria.

\section{Data collection and items}

Relevant data were extracted from the articles and entered onto a structured Microsoft ${ }^{\circ}$ Excel database. The information sought included: (i) Characteristics of the study patients (ii) Type of study and country (iii) Prevalence of hypertension (iv) Anti-hypertensive agents used (v) Achievement of target blood pressure.

\section{Assessment of risk of bias}

The risk of bias was assessed using the tool developed by Agency for Healthcare Research and Quality (AHRQ) [14] (Please see Additional file 2). This allowed systematic review of different potential sources of bias for each study type.

\section{Risk of bias}

The risk of bias for each study is summarised in Table 1.

\section{Method of synthesis}

Having extracted the data from the selected articles, the combined data was analysed to test whether there has been any change in treatment patterns over time using regression analysis. Where needed data from the articles were transformed to facilitate comparison of data.

\section{Results}

4079 citations were identified initially and, after applying limits and removing duplicates, this was reduced to 2627 citations. Of these 2583 articles were rejected after review of the abstract demonstrated that they did not meet the eligibility criteria. The full text of the remaining 43 articles was then reviewed in detail. 31 of these articles were then discarded after this review revealed that they were ineligible. One additional article was identified by review of the included articles' bibliographies which met the eligibility 
Table 1 Risk of bias

\begin{tabular}{|c|c|c|c|c|c|c|c|c|c|c|c|}
\hline \multirow[t]{2}{*}{ Source } & \multicolumn{4}{|c|}{ Selection bias } & \multirow{2}{*}{$\begin{array}{c}\begin{array}{c}\text { Performance } \\
\text { bias }\end{array} \\
\text { Outcome } \\
\text { assessors } \\
\text { blinded? }\end{array}$} & \multirow{2}{*}{$\begin{array}{c}\text { Attrition bias } \\
\text { Diagnosis } \\
\text { defined with } \\
\text { valid and } \\
\text { reliable } \\
\text { measures? }\end{array}$} & \multicolumn{3}{|c|}{ Detection bias } & \multirow{2}{*}{$\begin{array}{l}\begin{array}{c}\text { Publication } \\
\text { bias }\end{array} \\
\begin{array}{c}\text { Suspicion of } \\
\text { publication } \\
\text { bias? }\end{array}\end{array}$} & \multirow[t]{2}{*}{$\begin{array}{l}\text { Included in } \\
\text { synthesis? }\end{array}$} \\
\hline & $\begin{array}{l}\text { Inclusion/exclusion } \\
\text { criteria applied } \\
\text { uniformly? }\end{array}$ & $\begin{array}{l}\text { Confounding } \\
\text { accounted } \\
\text { for? }\end{array}$ & $\begin{array}{c}\text { Concurrent } \\
\text { intervention } \\
\text { accounted for? }\end{array}$ & $\begin{array}{l}\text { Missing data } \\
\text { handling? }\end{array}$ & & & $\begin{array}{l}\text { Outcomes } \\
\text { defined with } \\
\text { valid and } \\
\text { reliable } \\
\text { measures? }\end{array}$ & $\begin{array}{l}\text { Confounding } \\
\text { variables } \\
\text { assessed? }\end{array}$ & $\begin{array}{c}\text { Outcomes } \\
\text { pre-specified? }\end{array}$ & & \\
\hline $\begin{array}{l}\text { Amoo } \\
\text { et al. [25] }\end{array}$ & Yes & Yes & N/A & N/A & N/A & Yes & N/A & Yes & Yes & No & Yes \\
\hline $\begin{array}{l}\text { Andersen } \\
\text { et al. [26] }\end{array}$ & Yes & Yes & $\mathrm{N} / \mathrm{A}$ & $\mathrm{N} / \mathrm{A}$ & No & Yes & Yes & Yes & Yes & No & Yes \\
\hline $\begin{array}{l}\text { Davies } \\
\text { et al. [17] }\end{array}$ & Yes & Yes & N/A & Yes & No & Yes & Yes & Yes & Yes & No & Yes \\
\hline $\begin{array}{l}\text { Duron } \\
\text { et al. [20] }\end{array}$ & Yes & Yes & N/A & N/A & No & Yes & Yes & Yes & Yes & No & Yes \\
\hline $\begin{array}{l}\text { Hanon } \\
\text { et al. [19] }\end{array}$ & Yes & Yes & N/A & N/A & No & Yes & Yes & Yes & Yes & No & Yes \\
\hline $\begin{array}{l}\text { Imfeld } \\
\text { et al. [18] }\end{array}$ & Yes & Yes & N/A & Yes & No & Yes & Yes & Yes & Yes & No & Yes \\
\hline $\begin{array}{l}\text { Löppönen } \\
\text { et al. [23] }\end{array}$ & Yes & Yes & N/A & No & No & Yes & Yes & Yes & Yes & No & Yes \\
\hline $\begin{array}{l}\text { Múther } \\
\text { et al. [24] }\end{array}$ & Yes & Yes & N/A & $\begin{array}{l}\text { Yes - Missing } \\
\text { data for PWD }\end{array}$ & No & Yes & Yes & Yes & Yes & No & Yes \\
\hline $\begin{array}{l}\text { Poon } \\
\text { et al. [15] }\end{array}$ & Yes & Yes & N/A & Yes - excluded & No & Yes & Yes & Yes & Yes & No & Yes \\
\hline $\begin{array}{l}\text { Richards } \\
\text { et al. [16] }\end{array}$ & Yes & Yes & N/A & N/A & No & Yes & Yes & Yes & Yes & No & Yes \\
\hline $\begin{array}{l}\text { Rockwood } \\
\text { et al. [22] }\end{array}$ & Yes & Yes & N/A & Yes* $^{*}$ & No & Yes & Yes & Yes & Yes & No & Yes \\
\hline $\begin{array}{l}\text { Vale } \\
\text { et al. [21] }\end{array}$ & Yes & Yes & N/A & $\begin{array}{l}\text { Yes } 58.8 \% \\
\text { enrolled }\end{array}$ & No & Yes & Yes & Yes & Yes & No & Yes \\
\hline $\begin{array}{l}\text { Zhu } \\
\text { et al. [27] }\end{array}$ & Yes & Yes & N/A & N/A & No & Yes & Yes & Yes & Yes & No & Yes \\
\hline
\end{tabular}

*11.4\% not contactable, $27.9 \%$ refused (community) $3.2 \%$ not contactable, $18.3 \%$ refused (institutions). 
criteria. In total, therefore, 13 articles were included in the review (Figure 1).

\section{Characteristics of studies}

The characteristics of individual studies are summarised in Table 2. Of the 13 articles three reported studies which were conducted in the USA $[15,16]$, two each in the UK $[17,18]$ and France $[19,20]$ and one each in Brazil [21], Canada [22], Finland [23], Germany [24], Nigeria [25] and Norway [26].

\section{Methodology}

All 13 studies were observational studies. 11 were crosssectional and four of these were case-controlled $[17,18,24,26]$. The remaining two were cohort studies $[20,27]$. Of the 11 cross-sectional studies, six gathered data prospectively and five did so retrospectively $[15,17,18,24,25]$. Of the five retrospective studies the two UK studies and the German study used databases built using data held by primary care doctors $[17,18,24]$, and the remaining two retrospectively analysed digital and hard copy hospital data $[15,25]$.

All of the studies described their sampling method. Six studies invited routine attendees to their clinic or hospital to take part in their study [15,19-21,25,27], three studies used information from primary care databases to identify participants $[17,18,24]$, and four conducted population surveys $[16,22,23,26]$.

\section{Participants}

15,921 people with hypertension out of a total population of 23,804 were studied.

\section{Objectives}

The objectives of the studies varied. Three set out to describe the clinical profile, including information on demographics, comorbidities and medications, of patients with dementia $[21,25,27]$. Four studies aimed to compare comorbidities and medication use between those with and without dementia $[18,23,24,26]$, while one aimed to look specifically at treatment in those with vascular cognitive

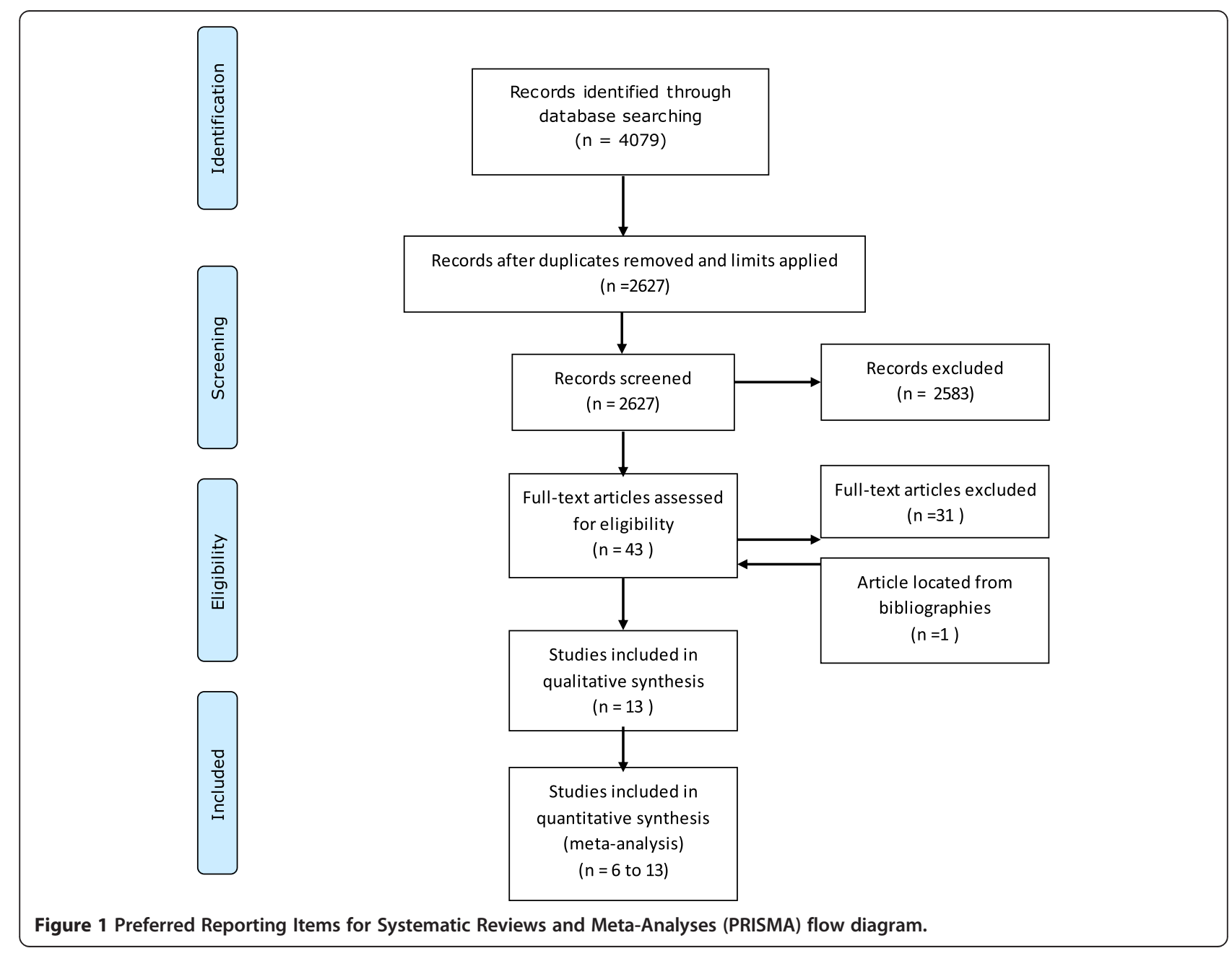


Table 2 Summary of the studies' characteristics

\begin{tabular}{|c|c|c|c|c|c|c|c|c|c|c|}
\hline $\begin{array}{l}\text { Source (year } \\
\text { published) }\end{array}$ & Type of study & $\begin{array}{l}\text { Number } \\
\text { with o } \\
\text { and suk }\end{array}$ & $\begin{array}{l}\text { eople } \\
\text { ntia } \\
\text { es (\%) }\end{array}$ & $\begin{array}{c}\text { Number with } \\
\text { HTN (\%) }\end{array}$ & $\begin{array}{l}\text { Mean age } \\
\text { (SD/range) }\end{array}$ & Location & Country & $\begin{array}{l}\text { Identification of } \\
\text { hypertensive pts }\end{array}$ & BP measured & Selection method \\
\hline \multirow{5}{*}{$\begin{array}{l}\text { Amoo } \\
\text { et al. [25] }\end{array}$} & Cross-sectional & \multicolumn{2}{|c|}{108} & \multirow[t]{5}{*}{$39(36.1)$} & \multirow[t]{5}{*}{70} & \multirow{5}{*}{$\begin{array}{l}\text { Neuro-psychiatric } \\
\text { Hospital }\end{array}$} & \multirow[t]{5}{*}{ Nigeria } & \multirow[t]{5}{*}{$\mathrm{BP}>140 / 90$} & \multirow[t]{5}{*}{ No } & \multirow{5}{*}{$\begin{array}{l}\text { All attendees with a } \\
\text { diagnosis of dementia } \\
\text { over a } 10 \text { year period }\end{array}$} \\
\hline & \multirow{4}{*}{$\begin{array}{l}\text { Retrospective } \\
\text { review of hospital } \\
\text { records }\end{array}$} & $A D$ & 57 & & & & & & & \\
\hline & & $\mathrm{VaD}$ & 17 & & & & & & & \\
\hline & & Mixed & 4 & & & & & & & \\
\hline & & Unsp. & 22 & & & & & & & \\
\hline \multirow{5}{*}{$\begin{array}{l}\text { Andersen } \\
\text { et al. [26] }\end{array}$} & Cross-sectional & \multicolumn{2}{|c|}{187} & \multirow[t]{5}{*}{$102(54.5)$} & \multirow[t]{5}{*}{$80.9(7)$} & \multirow{5}{*}{$\begin{array}{l}76 \% \text { community } \\
\text { dwelling, } 24 \% \text { in } \\
\text { long-term care }\end{array}$} & \multirow[t]{5}{*}{ Norway } & \multirow{5}{*}{$\begin{array}{l}\text { Self-reported } \\
\text { medical history }\end{array}$} & \multirow[t]{5}{*}{ No } & \multirow{5}{*}{$\begin{array}{l}\text { Recent diagnosis of } \\
\text { dementia and/or } \\
\text { population screening. } \\
\text { Randomly selected } \\
\text { control group }\end{array}$} \\
\hline & \multirow[t]{4}{*}{ Case controlled } & $A D$ & 100 & & & & & & & \\
\hline & & $\mathrm{VaD}$ & 0 & & & & & & & \\
\hline & & Mixed 0 & & & & & & & & \\
\hline & & Unsp. 0 & & & & & & & & \\
\hline \multirow{5}{*}{$\begin{array}{l}\text { Davies } \\
\text { et al. [17] }\end{array}$} & Cross-sectional & \multicolumn{2}{|c|}{20,021} & \multirow[t]{5}{*}{$9197(46)$} & \multirow[t]{5}{*}{$82.2(7)$} & \multirow{5}{*}{$\begin{array}{c}\text { UK General } \\
\text { Practice Research } \\
\text { Database (GPRD) }\end{array}$} & \multirow[t]{5}{*}{ UK } & \multirow{5}{*}{$\begin{array}{l}\text { Having ever } \\
\text { taken an } \\
\text { antihypertensive } \\
\text { for } 6 \text { months }\end{array}$} & \multirow[t]{5}{*}{ No } & \multirow{5}{*}{$\begin{array}{l}\text { Database. (Read } \\
\text { codes for probable } \\
\text { AD, possible AD, VaD } \\
\text { and unspecified / } \\
\text { other dementia } \\
\text { searched) }\end{array}$} \\
\hline & Case-controlled & $A D$ & 63 & & & & & & & \\
\hline & & $\mathrm{VaD}$ & 24 & & & & & & & \\
\hline & Retrospective & Mixed & 0 & & & & & & & \\
\hline & & Unsp. & 13 & & & & & & & \\
\hline Duron & Cohort & & & $221(68.8)$ & $78.1(6)$ & Memory Clinic & France & $B P>140 / 90$ & Yes & All patients diagnosed \\
\hline el al. [2U] & & $A D$ & 100 & & & & & & & $\begin{array}{l}\text { Witn Alznelmers } \\
\text { disease and on anti- }\end{array}$ \\
\hline & & $\mathrm{VaD}$ & 0 & & & & & & & cholinesterase \\
\hline & & Mixed & 0 & & & & & & & \\
\hline & & Unsp. & 0 & & & & & & & \\
\hline Hanon & Cross-sectional & & & $609(100)$ & $80.1(70-86)$ & Community & France & $\mathrm{BP}>140 / 90$ or & Yes & Consecutive \\
\hline 19] & & $A D$ & 86 & & & $\begin{array}{l}\text { dwellers attendıng } \\
\text { a memory clinic }\end{array}$ & & $\begin{array}{l}\text { takıng an } \\
\text { antihypertensive }\end{array}$ & & \\
\hline & & $\mathrm{VaD}$ & 14 & & & & & & & \\
\hline & & Mixed & 0 & & & & & & & \\
\hline & & Unsp. & 0 & & & & & & & \\
\hline Imfeld & Cross-sectional & & & $4926(42.7)$ & - & UK General & UK & Recorded & No & Database. (Read \\
\hline el al. [18] & Case controlled & $A D$ & 61 & & & Database (GPRD) & & DSIS & & $\begin{array}{l}\text { Codes } \text { and unspecified } \\
\text { and }\end{array}$ \\
\hline & Retrocnective & VaD & 39 & & & & & & & $\begin{array}{c}\text { dementia }+ \text { selection } \\
\text { algorithm) }\end{array}$ \\
\hline
\end{tabular}


Table 2 Summary of the studies' characteristics (Continued)

\begin{tabular}{|c|c|c|c|c|c|c|c|c|c|c|}
\hline \multirow{5}{*}{$\begin{array}{l}\text { Löppönen } \\
\text { et al. [23] }\end{array}$} & Cross-sectional & \multicolumn{2}{|c|}{94} & \multirow[t]{5}{*}{$48(51.1)$} & \multirow[t]{5}{*}{$84.4(5.7)$} & \multirow[t]{5}{*}{ Population based } & \multirow[t]{5}{*}{ Finland } & \multirow{5}{*}{$\begin{array}{c}\text { Recorded } \\
\text { diagnosis or BP } \\
>160 / 100\end{array}$} & \multirow[t]{5}{*}{ Yes } & \multirow{5}{*}{$\begin{array}{c}\text { All residents } \\
>65 \text { years of age, of } \\
\text { Lieto, were invited to } \\
\text { take part ( } 82 \% \text { took } \\
\text { part) }\end{array}$} \\
\hline & Population based & $A D$ & 43 & & & & & & & \\
\hline & & VaD & 37 & & & & & & & \\
\hline & & Mixed & 0 & & & & & & & \\
\hline & & Unsp. & 20 & & & & & & & \\
\hline \multirow{5}{*}{$\begin{array}{l}\text { Múther } \\
\text { et al. [24] }\end{array}$} & Cross-sectional & \multicolumn{2}{|c|}{216} & \multirow[t]{5}{*}{$181(83.8)$} & \multirow[t]{5}{*}{$82.7(6.2)$} & \multirow[t]{5}{*}{ GP database } & \multirow[t]{5}{*}{ Germany } & \multirow{5}{*}{$\begin{array}{l}\text { Recorded } \\
\text { diagnosis }\end{array}$} & \multirow[t]{5}{*}{ No } & \multirow{5}{*}{$\begin{array}{c}16 \text { of } 25 \text { invited } \\
\text { teaching GP practises. } \\
\text { Patients with a } \\
\text { recorded diagnosis of } \\
\text { dementia and one of } \\
\text { HTN, DM, } \\
\text { hyperlipidaemia }\end{array}$} \\
\hline & Case controlled & $A D$ & 0 & & & & & & & \\
\hline & & $\mathrm{VaD}$ & 0 & & & & & & & \\
\hline & Retrospective & Mixed & 0 & & & & & & & \\
\hline & & Unsp. & 100 & & & & & & & \\
\hline \multirow{5}{*}{$\begin{array}{l}\text { Poon } \\
\text { et al. [15] }\end{array}$} & Cross-sectional & \multicolumn{2}{|c|}{304} & \multirow[t]{5}{*}{$304(100)$} & \multirow[t]{5}{*}{78.1} & \multirow{5}{*}{$\begin{array}{l}\text { Outpatient } \\
\text { attendees VA } \\
\text { medical centre } \\
\text { clinics. }\end{array}$} & \multirow[t]{5}{*}{ USA } & \multirow{5}{*}{$\begin{array}{l}\text { Recorded } \\
\text { diagnosis }\end{array}$} & \multirow[t]{5}{*}{ No } & \multirow{5}{*}{$\begin{array}{c}\text { Recorded diagnoses } \\
\text { of both HTN and } \\
\text { dementia. }\end{array}$} \\
\hline & Retrospective & $A D$ & 60 & & & & & & & \\
\hline & & $\mathrm{VaD}$ & 35 & & & & & & & \\
\hline & & Mixed & 4 & & & & & & & \\
\hline & & Unsp. & 2 & & & & & & & \\
\hline \multirow{5}{*}{$\begin{array}{l}\text { Richards } \\
\text { et al. [16] }\end{array}$} & Cross-sectional & \multicolumn{2}{|c|}{65} & $37(56.9)$ & $81.4(6.4)$ & Urban dwellers & USA & Recorded & No & Random sample of a \\
\hline & & $A D$ & 75 & & & & & Jnosis & & from 29 contiguous \\
\hline & & VaD & 0 & & & & & & & census tracts. \\
\hline & & Mixed & 0 & & & & & & & \\
\hline & & Unsp. & 25 & & & & & & & \\
\hline Rockwood & Cross sectional & & 792 & $281(35.5)$ & - & $\begin{array}{c}\text { Survey of } \\
\text { Surve }\end{array}$ & Canada & Recorded & Yes & Stratified \\
\hline$[\angle 2]$ & & $A D$ & 57 & & & and community & & & & sample across the \\
\hline & & VaD & 26 & & & dwelling older & & & & country \\
\hline & & Mixed & 17 & & & & & & & \\
\hline & & Unsp. & 0 & & & & & & & \\
\hline $\begin{array}{l}\text { Vale } \\
\text { et al } 5211\end{array}$ & Cross-sectional & & 186 & $86(46.2)$ & $67.4(13.21)$ & Behavioural & Brazil & Recorded & No & $\begin{array}{l}\text { All first attendees } \\
\text { between } 1997\end{array}$ \\
\hline el al. $[\angle T]$ & & $A D$ & 31 & & & Outpatients Clinic. & & & & 1999 were invited to \\
\hline & & VaD & 19 & & & $\begin{array}{l}\text { Tertiary referral } \\
\text { centre }\end{array}$ & & & & take part \\
\hline & & Mixed & 9 & & & & & & & \\
\hline & & Unsp. & 41 & & & & & & & \\
\hline
\end{tabular}


Table 2 Summary of the studies' characteristics (Continued)

\begin{tabular}{|c|c|c|c|c|c|c|c|c|c|c|}
\hline \multirow[t]{5}{*}{ Zhu et al. [27] } & \multirow[t]{5}{*}{ Cohort } & \multicolumn{2}{|c|}{201} & \multirow[t]{5}{*}{71 (35.5) } & \multirow[t]{5}{*}{$76(8.1)$} & \multirow{5}{*}{$\begin{array}{l}84 \% \text { community } \\
\text { dwelling, } 16 \% \text { in } \\
\text { long-term care. }\end{array}$} & \multirow[t]{5}{*}{ USA } & \multirow{5}{*}{$\begin{array}{l}\text { Recorded } \\
\text { diagnosis }\end{array}$} & \multirow[t]{5}{*}{ No } & \multirow{5}{*}{$\begin{array}{c}\text { Consecutive } \\
\text { outpatient attendees; } \\
\text { Referrals; some long- } \\
\text { term care residents } \\
\text { MMSE > } 16 \text {, advocate } \\
\text { available. }\end{array}$} \\
\hline & & $A D$ & 100 & & & & & & & \\
\hline & & $\mathrm{VaD}$ & 0 & & & & & & & \\
\hline & & Mixed & 0 & & & & & & & \\
\hline & & Unsp. & 0 & & & & & & & \\
\hline
\end{tabular}


Table 3 Summary of the studies' findings

\begin{tabular}{|c|c|c|c|c|c|c|c|c|}
\hline \multirow{3}{*}{$\begin{array}{l}\text { Source (year published) } \\
\text { Amoo et al. [25] }\end{array}$} & \multirow{2}{*}{$\begin{array}{c}\text { Prevalence } \\
\text { of HTN (\%) } \\
36\end{array}$} & \multirow{2}{*}{$\begin{array}{c}\text { Sex (\%) } \\
47 \mathrm{M}\end{array}$} & \multicolumn{2}{|c|}{$\begin{array}{c}\text { Comorbidities } \\
\text { (\%) }\end{array}$} & \multicolumn{2}{|c|}{$\begin{array}{l}\text { Antihypertensive } \\
\text { types (\%) }\end{array}$} & \multirow{3}{*}{$\begin{array}{c}\text { Treated (\%) } \\
108 \text { (some on } \\
\text { antihypertensives for } \\
\text { other diagnoses) }\end{array}$} & \multirow{2}{*}{$\begin{array}{c}\begin{array}{c}\text { Effectiveness } \\
\text { (meets targe } \\
<140 / 90)\end{array} \\
-\end{array}$} \\
\hline & & & Heart failure & - & ACEi/ARB & - & & \\
\hline & & $53 \mathrm{~F}$ & $\mathrm{IHD}$ & - & Diuretic & - & & \\
\hline & & & DM & 6 & C C Blockers & - & & \\
\hline & & & CVD & 12 & $\beta$-Blockers & - & & \\
\hline & & & & & Other & - & & \\
\hline \multirow[t]{5}{*}{ Andersen et al. [26] } & 55 & $40 \mathrm{M}$ & Heart failure & - & ACEi/ARB & - & 125 (some on & - \\
\hline & & $60 \mathrm{~F}$ & $\mathrm{HD}$ & 40 & Diuretic & - & $\begin{array}{l}\text { antihypertensives for } \\
\text { other diagnoses) }\end{array}$ & \\
\hline & & & $\mathrm{DM}$ & 11 & C C Blockers & - & & \\
\hline & & & CVD & 18 & $\beta$-Blockers & - & & \\
\hline & & & & & Other & - & & \\
\hline \multirow[t]{5}{*}{ Davies et al. [17] } & 46 & $33 \mathrm{M}$ & Heart failure & - & ACEi/ARB & 40 & 100 (population selected & - \\
\hline & & $67 \mathrm{~F}$ & $\mathrm{HD}$ & 34 & Diuretic & 50 & $\begin{array}{c}\text { to be on an } \\
\text { antihypertensive) }\end{array}$ & \\
\hline & & & DM & 15 & C C Blockers & 42 & & \\
\hline & & & CVD & 26 & $\beta$-Blockers & 41 & & \\
\hline & & & & & Other & 10 & & \\
\hline \multirow[t]{5}{*}{ Duron et al. [20] } & 69 & $32 \mathrm{M}$ & Heart failure & - & ACEi/ARB & 37 & 78 & - \\
\hline & & $68 \mathrm{~F}$ & $\mathrm{IHD}$ & 26 & Diuretic & 30 & & \\
\hline & & & DM & 9 & C C Blockers & 29 & & \\
\hline & & & CVD & - & $\beta$-Blockers & 39 & & \\
\hline & & & & & Other & 6 & & \\
\hline \multirow[t]{5}{*}{ Hanon et al. [19] } & 100 & $28 \mathrm{M}$ & Heart failure & - & ACEi/ARB & - & 55 & - \\
\hline & & $72 \mathrm{~F}$ & $\mathrm{IHD}$ & - & Diuretic & - & & \\
\hline & & & DM & - & C C Blockers & - & & \\
\hline & & & CVD & - & $\beta$-Blockers & - & & \\
\hline & & & & & Other & - & & \\
\hline \multirow[t]{5}{*}{ Imfeld et al. [18] } & 43 & $35 \mathrm{M}$ & Heart failure & 9 & ACEi/ARB & 45 & & - \\
\hline & & $65 \mathrm{~F}$ & $\mathrm{IHD}$ & 22 & Diuretic & 90 & & \\
\hline & & & DM & 11 & C C Blockers & 45 & & \\
\hline & & & CVD & - & $\beta$-Blockers & 45 & & \\
\hline & & & & & Other & - & & \\
\hline \multirow[t]{5}{*}{ Löppönen et al. [23] } & 51 & $31 \mathrm{M}$ & Heart failure & 23 & ACEi/ARB & 12 & 85 & - \\
\hline & & $69 \mathrm{~F}$ & $\mathrm{IHD}$ & 57 & Diuretic & 46 & & \\
\hline & & & DM & 18 & C C Blockers & 27 & & \\
\hline & & & CVD & 37 & $\beta$-Blockers & 15 & & \\
\hline & & & & & Other & - & & \\
\hline \multirow[t]{5}{*}{ Múther et al. [24] } & 84 & $23 \mathrm{M}$ & Heart failure & - & ACEi/ARB & - & 85 & - \\
\hline & & $77 \mathrm{~F}$ & IHD & - & Diuretic & - & & \\
\hline & & & DM & - & C C Blockers & - & & \\
\hline & & & CVD & - & $\beta$-Blockers & - & & \\
\hline & & & & & Other & - & & \\
\hline \multirow[t]{3}{*}{ Poon et al. [15] } & 100 & $98 \mathrm{M}$ & Heart failure & 11 & ACEi/ARB & 59 & \multirow{3}{*}{$\begin{array}{l}100(2.95) \text { (population } \\
\text { selected to be on an } \\
\text { antihypertensive) }\end{array}$} & 55 \\
\hline & & $2 F$ & $\mathrm{IHD}$ & 31 & Diuretic & 57 & & \\
\hline & & & DM & 32 & C C Blockers & 44 & & \\
\hline
\end{tabular}


Table 3 Summary of the studies' findings (Continued)

\begin{tabular}{|c|c|c|c|c|c|c|c|c|}
\hline & & & CVD & 19 & $\beta$-Blockers & 40 & & \\
\hline & & & & & Other & 20 & & \\
\hline \multirow[t]{5}{*}{ Richards et al. [16] } & 57 & $35 \mathrm{M}$ & Heart failure & - & ACEi/ARB & 25 & 65 & - \\
\hline & & $65 \mathrm{~F}$ & $\mathrm{IHD}$ & - & Diuretic & 83 & & \\
\hline & & & DM & 18 & C C Blockers & 42 & & \\
\hline & & & CVD & - & $\beta$-Blockers & 8 & & \\
\hline & & & & & Other & 13 & & \\
\hline \multirow[t]{5}{*}{ Rockwood et al. [22] } & 35 & $29 M$ & Heart failure & - & ACEi/ARB & - & 53 & - \\
\hline & & $71 \mathrm{~F}$ & $\mathrm{IHD}$ & - & Diuretic & - & & \\
\hline & & & DM & - & C C Blockers & - & & \\
\hline & & & CVD & - & $\beta$-Blockers & - & & \\
\hline & & & & & Other & - & & \\
\hline \multirow[t]{5}{*}{ Vale et al. [21] } & 46 & $59 M$ & Heart failure & - & ACEi/ARB & - & 88 & - \\
\hline & & $41 \mathrm{~F}$ & $\mathrm{IHD}$ & - & Diuretic & - & & \\
\hline & & & $\mathrm{DM}$ & - & C C Blockers & - & & \\
\hline & & & CVD & - & $\beta$-Blockers & - & & \\
\hline & & & & & Other & - & & \\
\hline \multirow[t]{5}{*}{ Zhu et al. [27] } & 35 & Not available & Heart failure & - & ACEi/ARB & - & 48 & - \\
\hline & & & $\mathrm{IHD}$ & 6 & Diuretic & - & & \\
\hline & & & DM & 11 & C C Blockers & - & & \\
\hline & & & CVD & - & $\beta$-Blockers & - & & \\
\hline & & & & & Other & - & & \\
\hline
\end{tabular}

$[-=$ data not available $]$.

impairment [22]. Two studies aimed to look at the association between antihypertensives and cognitive impairment $[16,17]$. Two set out to evaluate the effect of antihypertensive therapy on cognitive function $[19,20]$ and one study aimed to compare blood pressure control and medication between different ethnic groups [15].

\section{Individual study findings}

The findings of each individual study are summarised in Table 3.

\section{Synthesis of results}

Data were combined from each study where available.

\section{Characteristics of study participants}

The average age of the patients across the studies was 82 , with the majority (65\%) being female. Alzheimer's disease was the most common dementia subtype (63\%), followed by vascular dementia (30\%), unspecified dementia $(7 \%)$ and mixed dementia $(0.7 \%)$. The population had a high burden of co-morbidity with $27 \%$ having ischaemic heart disease, $26 \%$ cerebrovascular disease, $12.7 \%$ diabetes mellitus and $9.3 \%$ heart failure.

\section{Prevalence}

The prevalence of hypertension in people with dementia as reported by these studies varied between a minimum of $35 \%$ [27] and a maximum of $84 \%$ [24]. The mean prevalence of hypertension across the studies was $45 \%$ (SD 11\%). There was no change in the prevalence of hypertension over time when earlier and more recent studies were compared.

\section{Prescribing patterns}

Of the eight studies [16,19-24,27] which reported details of treatment between $48 \%$ and $85 \%$ of their participants were on at least one antihypertensive agent. Across all studies a mean of $73 \%$ were on at least one antihypertensive agent.

Diuretics (64\%, range 30\%-90\%) were most commonly used, while calcium channel blockers (43\%, range $27 \%$ $45 \%$ ), ACEi/ARBs (42\%, range $12 \%-59 \%$ ) and $\beta$-blockers (42\%, range $8 \%-45 \%$ ) were less common.

A higher proportion of the hypertensive care home population took ACEi / ARBs (correlation coefficient: 0.85, $\mathrm{R}^{2}=0.73, \mathrm{p}=0.031$ ), and calcium channel blockers (correlation coefficient: $0.82, \mathrm{R}^{2}=0.58, \mathrm{p}=0.048$ ) in later studies than in earlier studies, while the use of $\beta$-blockers and diuretics remained unchanged between later and earlier studies. 


\section{Number of antihypertensive agents and target blood pressure}

Two studies reported details of the number of antihypertensives used $[15,20]$. The mean number of antihypertensives was 2.4. Only one study reported on the achievement of target blood pressure [15], with 55\% achieving this. This study involved 304 people, almost all male, in a Veteran Affairs hospital.

\section{Discussion}

This review has demonstrated that hypertension is common in people with dementia and is treated in the majority. The prevalence of hypertension in people with dementia remained unchanged between older and more recent studies. Although diuretics were the most frequently prescribed antihypertensive drug, ACE inhibitors, ARBs and calcium channel blockers were prescribed more frequently in more recent studies. There was no change in the prescription of diuretics or $\beta$-blockers over this 13 year period. Only one study reported on achievement of target blood pressure, with just over half of individuals achieving this.

The studies reviewed included several large database studies, and so are likely to be representative of ordinary practice. However, they were carried out almost entirely within North America and Europe, and so the findings may not apply to countries with other health systems and prescribing habits such as in Asia or non-English speaking countries. Two of the studies reported whether participants had ever been on an antihypertensive drug rather than their current treatment. The inclusion of these data in the synthesis will have had the effect of increasing the apparent proportion on each antihypertensive class.

We are unaware of any previous systematic review looking at the treatment of hypertension in people with dementia. Similarly we are unaware of any specific guidance for the treatment of hypertension in people with dementia with which to compare these findings. Over the study period the JNC, along with others, issued a number of reports (V-VII) with changes in the generic guidance for the treatment of hypertension in older people. The rise over time in the use of ACE inhibitors / ARBs and calcium channel blockers is likely to reflect these changes in guidance. The fact that prevalence of hypertension did not change over time, despite lower blood pressure thresholds for diagnosis over time, raises the possibility that the true prevalence would have fallen over time had current, stricter, criteria for diagnosis being used throughout. It is not possible to comment from our findings whether this was the case, since several studies reported hypertension dichotomously as either present or absent using diagnostic criteria of the time, rather than presenting raw blood pressure data that we could re-analyse.

This review found no evidence that hypertension in people with dementia was not being treated. Whereas
$49 \%-63 \%$ of people in the general US population with hypertension were on treatment $[28,29]$, this review found that $73 \%$ of hypertensive people with dementia were on treatment. Side-effects are recognised to be a potent contributor to non-compliance in antihypertensive therapy $[30,31]$ and the higher rates of treatment raise the possibility that side effects of antihypertensive therapy in those with dementia may be either unrecognised or unreported, in which case the favourable risk to benefit ratio observed in trials of the non-frail may not apply. In addition with theoretical concerns that, with impaired cerebral autoregulation, this population are at increased risk of cerebral hypoperfusion [11] high rates of anti-hypertensive use, with the potential to exacerbate this, may not be ideal. Blood pressure falls as part of the natural history of dementia, starting prior to clinically apparent dementia [32] and it is intriguing that while most studies show that only $22-27 \%$ of the general hypertensive population achieve target blood pressures [28,29], 55\% reached target blood pressures in the one study which reported on this in people with dementia. A recent study looking at the incidence of frailty in older patients subject to polypharmacy suggested that the use of diuretics was associated with emergent frailty, whilst ACE-inhibitors were not [33]. Thus the gradual shift, over time, to greater prescription of ACE-inhibitors may be desirable. The persistent use of diuretics may not.

\section{Conclusion}

This review suggests that people with dementia are not managed differently from patients without dementia despite their increased risk of adverse events and the decreased likelihood that they will be recognised or reported. The findings presented here will support further work to establish the risk-benefit of antihypertensive treatment in patients with dementia and, if differing ratios are identified, to establish dementia-specific guidelines for management.

\section{Additional files}

Additional file 1: Search strategy Medline (Pubmed).

Additional file 2: Bias assessment tool.

\section{Abbreviations}

BP: Blood pressure.

\section{Competing interests}

The authors declare that they have no proprietary, financial, professional, or other personal competing interests of any nature or kind.

\section{Authors' contributions}

The literature search was designed and carried out by TJW, and the manuscript was drafted by TJW with contributions from JRG and ALG. The manuscript was critically revised by all three authors. All authors read and approved the final manuscript. 
Received: 1 November 2013 Accepted: 7 February 2014

Published: 12 February 2014

\section{References}

1. Cohen DL, Townsend RR: Update on pathophysiology and treatment of hypertension in the elderly. Curr Hypertens Rep 2011, 13(5):330-337.

2. Beckett NS, Peters R, Fletcher AE, Staessen JA, Liu L, Dumitrascu D, Stoyanovsky V, Antikainen RL, Nikitin Y, Anderson C, et al: Treatment of hypertension in patients 80 years of age or older. N Engl J Med 2008, 358(18):1887-1898.

3. Staessen JA, Fagard R, Thijs L, Celis H, Arabidze GG, Birkenhager WH, Bulpitt $C J$, de Leeuw PW, Dollery CT, Fletcher AE, et al: Randomised double-blind comparison of placebo and active treatment for older patients with isolated systolic hypertension. The Systolic Hypertension in Europe (Syst-Eur) Trial Investigators. Lancet 1997, 350(9080):757-764.

4. Prevention of stroke by antihypertensive drug treatment in older persons with isolated systolic hypertension. Final results of the Systolic Hypertension in the Elderly Program (SHEP). SHEP Cooperative Research Group. JAMA 1991, 265(24):3255-3264.

5. Lithell H, Hansson L, Skoog I, Elmfeldt D, Hofman A, Olofsson B, Trenkwalder P, Zanchetti A, Group SS: The Study on Cognition and Prognosis in the Elderly (SCOPE): principal results of a randomized double-blind intervention trial. J Hypertens 2003, 21(5):875-886.

6. Musini VM, Tejani AM, Bassett K, Wright JM: Pharmacotherapy for hypertension in the elderly. Cochrane Database Syst Rev 2009, 4, CD000028.

7. The fifth report of the Joint National Committee on Detection: Evaluation, and treatment of high blood pressure (JNC V). Arch Intern Med 1993, 153(2):154-183.

8. Chobanian AV, Bakris GL, Black HR, Cushman WC, Green LA, Izzo JL Jr, Jones DW, Materson BJ, Oparil S, Wright JT Jr, et al: The Seventh Report of the Joint National Committee on Prevention, Detection, Evaluation, and Treatment of High Blood Pressure: the JNC 7 report. JAMA 2003, 289(19):2560-2572.

9. Robertson DA, Savva GM, Kenny RA: Frailty and cognitive impairmentA review of the evidence and causal mechanisms. Ageing Res Rev 2013, 12(4):840-851.

10. Sonnesyn H, Nilsen DW, Rongve A, Nore S, Ballard C, Tysnes OB, Aarsland D: High prevalence of orthostatic hypotension in mild dementia. Dement Geriatr Cogn Disord 2009, 28(4):307-313.

11. Qiu C, Winblad B, Fratiglioni L: Low diastolic pressure and risk of dementia in very old people: a longitudinal study. Dement Geriatr Cogn Disord 2009, 28(3):213-219.

12. Gnjidic D, Hilmer SN, Blyth FM, Naganathan V, Waite L, Seibel MJ, McLachlan AJ, Cumming RG, Handelsman DJ, Le Couteur DG: Polypharmacy cutoff and outcomes: five or more medicines were used to identify community-dwelling older men at risk of different adverse outcomes. J Clin Epidemiol 2012, 65(9):989-995.

13. Morley JE: Systolic hypertension should Not Be treated in persons aged 80 and older until blood pressure is greater than $160 \mathrm{mmHg}$. J Am Geriatr Soc 2013, 61(7):1197-1198.

14. Viswanathan MAM, Berkman ND, Chang S, Hartling L, McPheeters LM, Santaguida PL, Shamliyan T, Singh K, Tsertsvadze A, Treadwell JR: Assessing the risk of bias of individual studies in systematic reviews of health care interventions. USA: DoHaHS: AHRQ; 2012.

15. Poon I, Lal LS, Ford ME, Braun UK: Racial/ethnic differences in blood pressure control and medication utilization in a cohort of older veterans with dementia. Am J Ther 2010, 17(1):34-41.

16. Richards SS, Emsley CL, Roberts J, Murray MD, Hall K, Gao S, Hendrie HC: The association between vascular risk factor-mediating medications and cognition and dementia diagnosis in a community-based sample of African-Americans. J Am Geriatr Soc 2000, 48(9):1035-1041.

17. Davies NM, Kehoe PG, Ben-Shlomo Y, Martin RM: Associations of antihypertensive treatments with Alzheimer's disease, vascular dementia, and other dementias. J Alzheimers Dis 2011, 26(4):699-708.

18. Imfeld P, Brauchli Pernus YB, Jick SS, Meier CR: Epidemiology, co-morbidities, and medication use of patients with Alzheimer's disease or vascular dementia in the UK. J Alzheimers Dis 2013, 35(3):565-573.

19. Hanon O, Pequignot R, Seux ML, Lenoir H, Bune A, Rigaud AS, Forette F, Girerd X: Relationship between antihypertensive drug therapy and cognitive function in elderly hypertensive patients with memory complaints. J Hypertens 2006, 24(10):2101-2107.
20. Duron E, Rigaud AS, Dubail D, Mehrabian S, Latour F, Seux ML, Hanon O: Effects of antihypertensive therapy on cognitive decline in Alzheimer's disease. Am J Hypertens 2009, 22(9):1020-1024.

21. Vale FA, Miranda SJ: Clinical and demographic features of patients with dementia attended in a tertiary outpatient clinic. Arq Neuropsiquiatr 2002, 60(3-A):548-552.

22. Rockwood K, Ebly E, Hachinski V, Hogan D: Presence and treatment of vascular risk factors in patients with vascular cognitive impairment. Arch Neurol 1997, 54(1):33-39.

23. Lopponen M, Raiha I, Isoaho R, Vahlberg T, Puolijoki H, Kivela SL: Dementia associates with undermedication of cardiovascular diseases in the elderly: a population-based study. Dement Geriatr Cogn Disord 2006, 22(2):132-141.

24. Muther J, Abholz HH, Wiese B, Fuchs A, Wollny A, Pentzek M: Are patients with dementia treated as well as patients without dementia for hypertension, diabetes, and hyperlipidaemia? Br J Gen Pract 2010, 60(578):671-674.

25. Amoo G, Akinyemi RO, Onofa LU, Akinyemi JO, Baiyewu O, Ogunlesi AO, Ogunniyi A: Profile of clinically-diagnosed dementias in a neuropsychiatric practice in Abeokuta, south-western Nigeria. Afr J Psychiatry 2011, 14(5):377-382.

26. Andersen F, Viitanen M, Halvorsen DS, Straume B, Engstad TA: Co-morbidity and drug treatment in Alzheimer's disease. A cross sectional study of participants in the dementia study in northern Norway. BMC Geriatr 2011, 11:58

27. Zhu CW, Livote EE, Kahle-Wrobleski K, Scarmeas N, Albert M, Brandt J, Blacker D, Sano M, Stern Y: Utilization of antihypertensives, antidepressants, antipsychotics, and hormones in Alzheimer disease. Alzheimer Dis Assoc Disord 2011, 25(2):144-148.

28. Hajjar I, Kotchen JM, Kotchen TA: Hypertension: trends in prevalence, incidence, and control. Annu Rev Public Health 2006, 27:465-490.

29. National High Blood Pressure Education Program Working Group: National high blood pressure education program working group report on hypertension in the elderly. Hypertension 1994, 23(3):275-285

30. After the diagnosis: adherence and persistence with hypertension therapy. Am J Manag Care 2005, 11(13):S395-S399.

31. Bloom BS: Continuation of initial antihypertensive medication after 1 year of therapy. Clin Ther 1998, 20(4):671-681.

32. Qiu C, von Strauss E, Winblad B, Fratiglioni L: Decline in blood pressure over time and risk of dementia: a longitudinal study from the Kungsholmen project. Stroke 2004, 35(8):1810-1815.

33. Gnjidic D, Hilmer SN, Blyth FM, Naganathan V, Cumming RG, Handelsman DJ, McLachlan AJ, Abernethy DR, Banks E, Le Couteur DG: High-risk prescribing and incidence of frailty among older community-dwelling men. Clin Pharmacol Ther 2012, 91(3):521-528.

doi:10.1186/1471-2318-14-19

Cite this article as: Welsh et al:: The treatment of hypertension in people with dementia: a systematic review of observational studies. BMC Geriatrics 2014 14:19.

\section{Submit your next manuscript to BioMed Central and take full advantage of:}

- Convenient online submission

- Thorough peer review

- No space constraints or color figure charges

- Immediate publication on acceptance

- Inclusion in PubMed, CAS, Scopus and Google Scholar

- Research which is freely available for redistribution 